\title{
Estimation of EMI Impact by Cellular Radio on Implantable Cardiac Pacemakers in Elevator Using EMF Distributions Inside Human Body
}

\author{
Atsushi KITAGAWA ${ }^{\dagger}$, Student Member, Takashi HIKAGE ${ }^{\dagger a)}$, Member, $^{\text {Toshio NOJIMA }}{ }^{\dagger}$, Fellow, \\ Ally Y. SIMBA ${ }^{\dagger \dagger}$, and Soichi WATANABE ${ }^{\dagger \dagger}$, Members
}

\begin{abstract}
SUMMARY The purpose of this study is to estimate the possible effect of cellular radio on implantable cardiac pacemakers in elevators. We previously investigated pacemaker EMI in elevator by examining the Efield distribution of horizontal plane at the height of expected for implanted pacemakers inside elevators. In this paper, we introduce our method for estimating EMI impact to implantable cardiac pacemakers using EMF distributions inside the region of the human body in which pacemakers are implanted. Simulations of a human phantom in an elevator are performed and histograms are derived from the resulting EMF distributions. The computed results of field strengths are compared with a certain reference level determined from experimentally obtained maximum interference distance of implantable cardiac pacemakers. This enables us to carry out a quantitative evaluation of the EMI impact to pacemakers by cellular radio transmission. This paper uses a numerical phantom model developed based on an European adult male. The simulations evaluate EMI on implantable cardiac pacemakers in three frequency bands. As a result, calculated E-field strengths are sufficiently low to cause the pacemaker to malfunction in the region examined.

key words: implantable cardiac pacemakers, cellular radios, elevator, finite difference time domain method, numerical phantom model
\end{abstract}

\section{Introduction}

In recent years, mobile phone usage has extended to a wide range of environment such as places surrounded by conductive surfaces, e.g., train carriages, elevators, and airplanes. There is a concern that the use of cellular phones in elevators might cause high exposure levels due to the reflection and accumulation of cellular radio [1]-[3]. There have also been concerns that cellular radio can interfere with different types of devices and increased usage in multi-reflection environments has led to concern about the possible effect of electromagnetic interference (EMI) on implantable medical devices (IMDs).

Many experimental studies have been carried out in order to assess the EMI generated by cellular radio in freespace and experienced by IMDs including implantable cardiac pacemakers and implantable cardioverter defibrillators (ICDs) [4]-[6]. However, few studies have addressed the effect of EMI on IMDs in elevators. Precise and efficient

Manuscript received November 1, 2009.

Manuscript revised March 8, 2010.

$\dagger$ The authors are with Hokkaido University, Sapporo-shi, 0600814 Japan.

${ }^{\dagger}$ The authors are with National Institute of Information and Communications Technology, Koganei-shi, 184-8795 Japan.

a)E-mail: hikage@wtemc.ist_hokudai.ac.jp

DOI: 10.1587/transcom.E93.B.1839 methods of measuring the electric field (E-field) strength in different regions of multi-reflection environments have not been advanced due to the disturbed fields caused by the presence of measurement equipment and/or human bodies. Therefore, this is achieved by carrying out precise numerical simulations using the Finite-Difference-Time-Domain method [7], [8].

We have already carried out numerical simulations on homogeneous human phantom models and some elevator models to investigate the EMI impact to implantable cardiac pacemakers in elevators; we have examined the E-field distribution on freespace inside elevator [9], [10]. However, implantable cardiac pacemaker is put inside human body. Therefore, more practical investigation is required in realistic environment, that is inside of human body.

In this paper, we propose our method for estimating pacemaker EMI using EMF distributions inside the region of the human body into which pacemakers are implanted. The calculated E-field strength is normalized to a certain reference level determined from the experimentally obtained maximum interference distance [11] of implantable cardiac pacemakers. This enables us to carry out quantitative evaluations of the EMI impact to pacemakers by cellular radio transmission [12].

In order to examine the complex situations where human is present in elevators, our analysis considered a realistically-shaped and anatomically-correct human phantom model. A half-wavelength dipole antenna is used to represent a cellular radio operating in the $900 \mathrm{MHz}, 1500 \mathrm{MHz}$, and $2100 \mathrm{MHz}$ frequency bands. All calculations were run on a supercomputer. We compare previous method using homogeneous phantom model and proposing method using inhomogeneous phantom model. Additionally, pacemaker EMI is evaluated when the presence or absence of a pacemaker.

The following section explains the estimation method used to determine the EMI impact on pacemakers, focusing on the elevator model and the human phantom models used in the calculation. Next, we introduce our method for estimating EMI impact in a comparison to the previous method. EMI impact in elevators are then examined using the proposing method. Finally, the results are discussed. 


\section{Methods}

The numerical simulations were carried out by applying the finite-difference time-domain (FDTD) method. The FDTD method is a versatile and efficient tool for the solution of Maxwell's equations in complex structures. It can also treat problem spaces that contain lossy media such as the human body. Additionally, a histogram estimation method for determining the electric field strength was developed to deal with the complicated EMF distributions. Histograms are useful for carrying out complete estimations of the whole region that is being modeled.

In order to estimate the pacemaker EMI, the EMF values are plotted using histograms and compared a certain reference values determined from the experimentally obtained maximum interference distance of implantable cardiac pacemakers. If there is any region of the histogram that exceeds this reference value, the implication is that there is some possibility of the EMI negatively impacting the pacemaker. This is explained in more detail below.

The details of the FDTD analysis configurations are summarized in Table 1 . We used a half-wavelength dipole antenna to represent a cellular radio operating in $900 \mathrm{MHz}$, $1500 \mathrm{MHz}$ and $2100 \mathrm{MHz}$ bands. Perfectly matched layer (PML) [13] having 16 layers were placed at all boundaries of the FDTD problem space to absorb the radiated outgoing waves. The time step was set to fill the Courant condition. The time steps used in the simulations were $8.96 \mathrm{psec}$, $8.77 \mathrm{psec}$, and $8.50 \mathrm{psec}$ for $900,1500,2100 \mathrm{MHz}$, respectively. These time steps were set to improve absorption characteristics of PML for each frequency. In order to achieve simulated results for steady field distributions in the elevator for all three frequency bands, more than hundreds periods of the FDTD computation were carried out. Numbers of periods used in the simulations were 200 periods, 400 periods, and 500 periods, and the time duration of simulations were approximately 15 hours, 18 hours, and 18 hours for $900 \mathrm{MHz}, 1500 \mathrm{MHz}$, and $2100 \mathrm{MHz}$.

Table 1 Computation parameters.

\begin{tabular}{l||l}
\hline Cell size (cubic) & $\mathrm{d}=5 \mathrm{~mm}$ \\
\hline Total problem space & $444 \times 560 \times 552$ (cells) \\
\hline Elevator model & $324 \times 440 \times 432$ (cells) \\
\hline Absorbing B. C. & PML (16 layers) \\
\hline Frequency & $900 \mathrm{MHz}, 1500 \mathrm{MHz}, 2100 \mathrm{MHz}$ \\
\hline Number of period & $\begin{array}{l}200(900 \mathrm{MHz}), 400(1500 \mathrm{MHz}), \\
500(2100 \mathrm{MHz})\end{array}$ \\
\hline & $\begin{array}{l}\text { Body: Perfect Electric Conductor } \\
\text { Openings: } \\
\text { Elevator model }\end{array}$ \\
& $-\quad$ Rectangular: $300 \times 600 \mathrm{~mm}$ \\
\hline Human phantom model & $\begin{array}{l}\text { Inhomogeneous } \\
\text { Realistic shape }\end{array}$ \\
\hline Required memory & 32 GB \\
\hline Cellular radio & Dipole antenna \\
\hline
\end{tabular}

\subsection{Elevator Model}

A 3-D representation of the numerical model of the elevator is shown in Fig. 1 [14]. This is the case in which only one passenger is present in the elevator. The dimensions are $2760 \mathrm{~mm} \times 2220 \mathrm{~mm} \times 2800 \mathrm{~mm}$ inclusive of the perfect matched layers (PML) and guard cells. The elevator's actual dimensions are $2160 \mathrm{~mm} \times 1620 \mathrm{~mm} \times 2200 \mathrm{~mm}$ (Length $\times$ Width $\times$ Height). The elevator model assumes that the physical properties of the elevator's body mirrors those of perfect electric conductor (PEC). In all models, the elevator door is closed. The openings in the roof of the elevator are the means by which cellular radios are able to propagate into and out of the elevator. The rectangular opening is $30 \mathrm{~cm} \times 60 \mathrm{~cm}$ and the circular opening has a diameter of $30 \mathrm{~cm}$.

\subsection{Human Phantom Model}

Figure 2 shows front and side-views of the human phantom model that was placed in the elevator. The human phantom model is an enhanced high-resolution magnetic resonance imaging whole-body human model taken from the Virtual

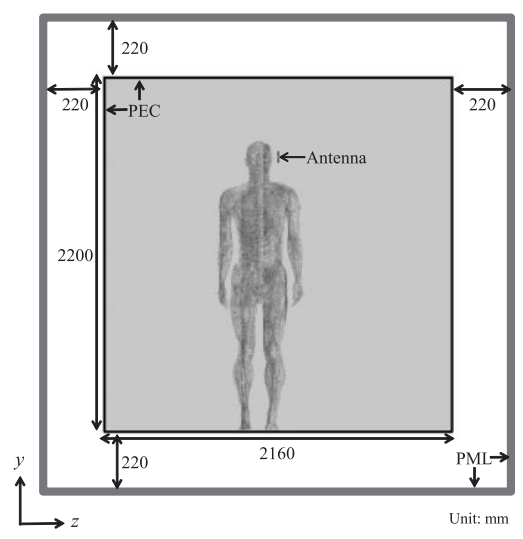

(a)

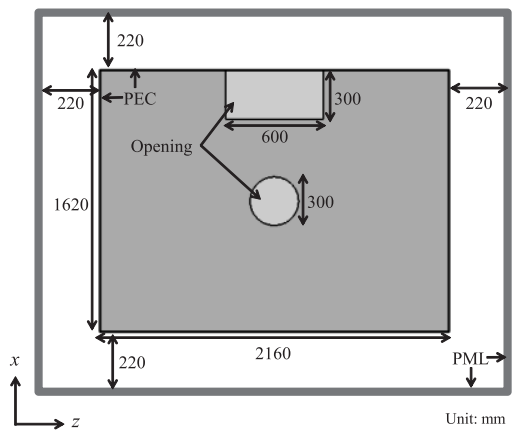

(b)

Fig. 1 Representation of the FDTD model of an elevator with a single user. (a) yz-plane (front view), (b) xz-plane (top view). 


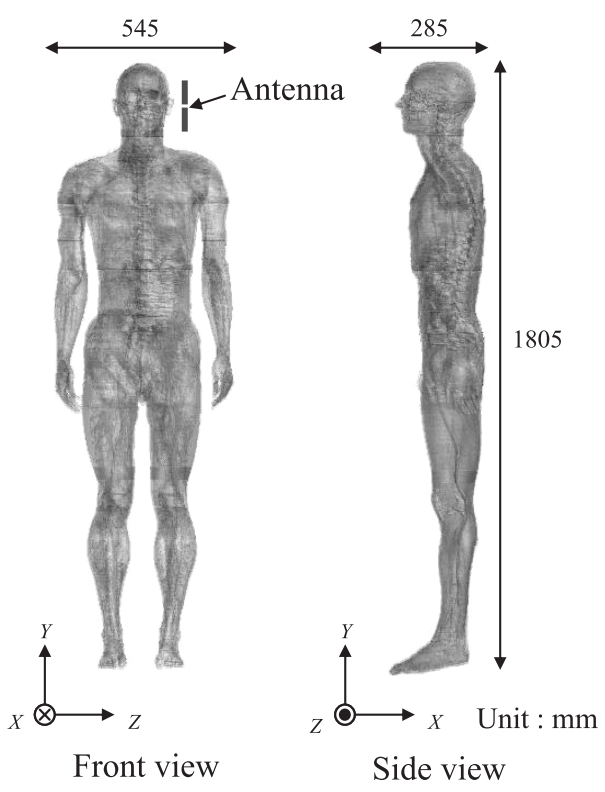

Fig. 2 Numerical phantom model.

Family Product series [15]. The phantom model has realistic shapes and is composed of 77 different tissues. The electric properties of the tissues were derived from [16]. Its height is about $180.5 \mathrm{~cm}$ while the shoulder-to-shoulder width is $54.5 \mathrm{~cm}$. The phantom's resolution is $5 \times 5 \times 5 \mathrm{~mm}^{3}$. The mobile phone is modeled as a half wavelength dipole antenna. The antenna is located $20 \mathrm{~mm}$ from the human phantom's head and is $1700 \mathrm{~mm}$ above the floor. The positions of the human phantom in the elevator were varied to observe the effect of distance from the wall of the elevator.

\subsection{Histogram Estimation Method for Pacemaker EMI}

Previously, we investigated pacemaker EMI impact by determining the E-field strength values on a horizontal plane inside the elevator; the reference level was the level that would cause pacemaker malfunction. In Fig. 3, an example of those E-field distributions inside the elevator is presented for the case of 1 user standing at the center of the elevator. The frequency bands are $900 \mathrm{MHz}, 1500 \mathrm{MHz}$, and $2100 \mathrm{MHz}$. The estimation plane is $1300 \mathrm{~mm}$ above the floor. This is the height at which most pacemakers are implanted. The resultant E-field distributions indicate a complicated environment since the problem space is a highly reflective.

In order to examine the situation in more practical, we propose a method that estimates EMI impact inside the phantom model. Figure 4, shows the position of an implantable cardiac pacemaker that has been implanted into a human torso. The connector from the cardiac pacemaker to the lead wires is also shown. This connector and cardiac pacemaker are set at a location just below the collarbone. Therefore, the area near the collar-bone, where most pacemakers are implanted, is examined. The electric field values in this region are compared against a ref-

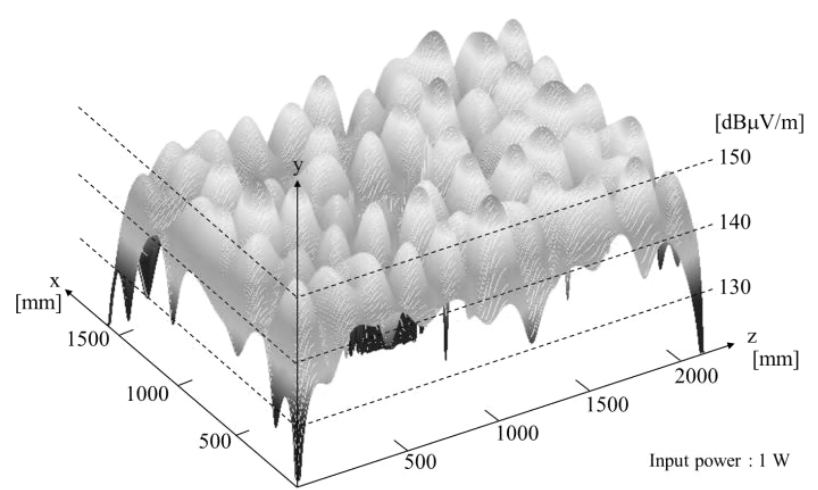

(a)

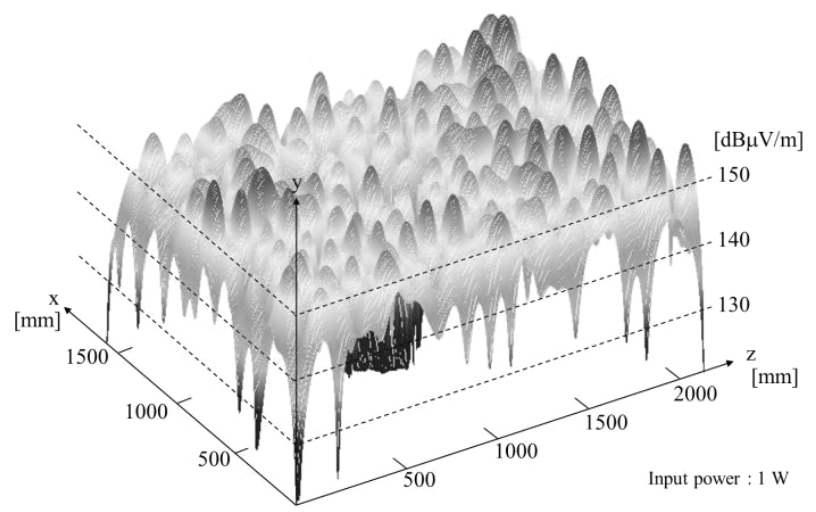

(b)

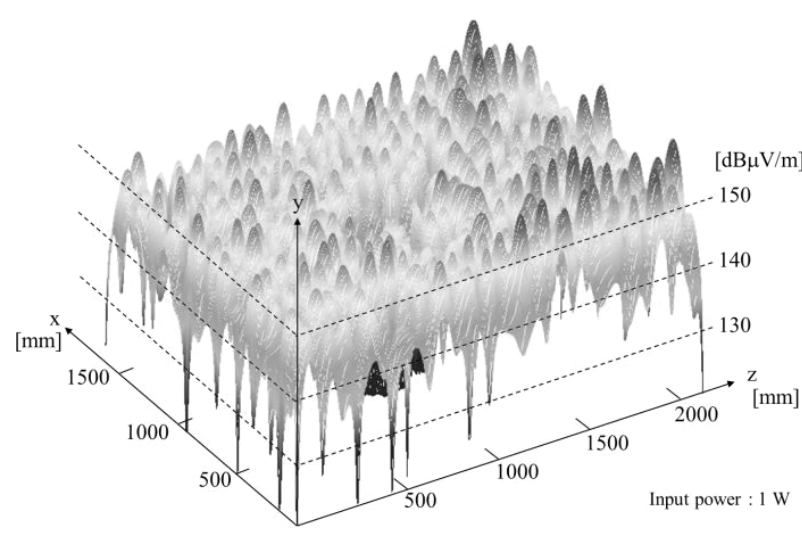

(c)

Fig. 3 Example of E-field Distribution at a height of $130 \mathrm{~cm}$ in elevator for (a) $900 \mathrm{MHz}$, (b) $1500 \mathrm{MHz}$, and (c) $2100 \mathrm{MHz}$.

erence level that might cause pacemaker malfunction. Figure 5 shows an example of the region considered. The region within the maximum interference distance from feeding point, which is explained in more detail below, is not examined. The volumes of the regions examined were approximately $310 \mathrm{~cm}^{3}, 320 \mathrm{~cm}^{3}, 320 \mathrm{~cm}^{3}$ for the frequencies of $900 \mathrm{MHz}, 1500 \mathrm{MHz}$, and $2100 \mathrm{MHz}$, respectively. The user position was changed from the center of the elevator to a corner of the elevator and the effect of this change was determined. Figure 6 shows different position of the phantom in the elevator. 


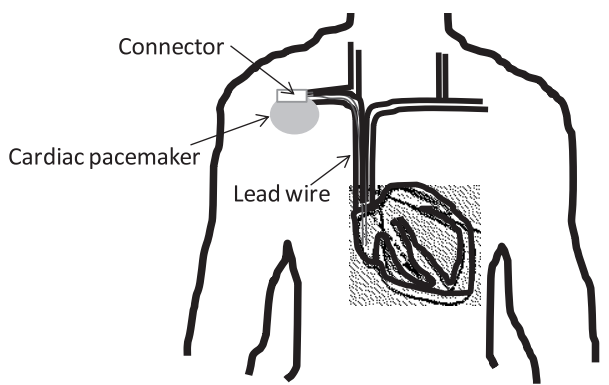

Fig. 4 Implantable cardiac pacemaker, connector, and lead wire.

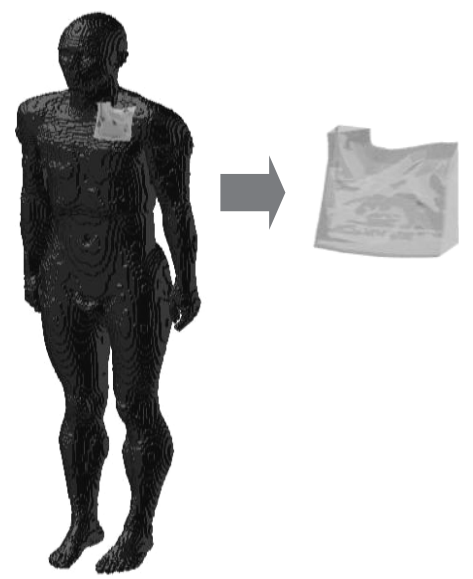

Fig. 5 Representation of the extracted region.

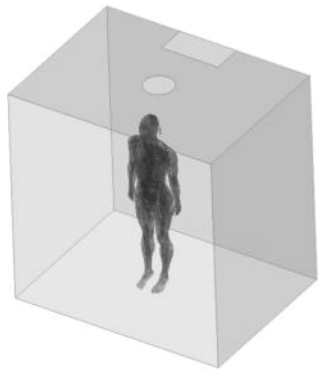

(a)

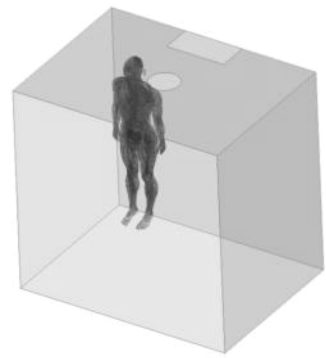

(b)
Fig. 6 Position of the phantom inside the elevator, (a) center, and (b) corner.

In addition, in order to estimate practical EMI impact, the simulations considered that the pacemaker was implanted inside a human body. The pacemaker was modeled as a metal box with dimension of $4 \mathrm{~cm} \times 4.5 \mathrm{~cm} \times 1 \mathrm{~cm}$. It was implanted inside the phantom body near the collar bone and 1 to $2 \mathrm{~cm}$ beneath the skin. Figure 7 shows the phantom model with the pacemaker. E-field strengths with and without the pacemaker were compared.

It is difficult to accurately estimate the values in the Efield distribution. Histogram estimation method is therefore used to plot the field strength values that exist throughout the area which examined.

In order to achieve our stated goal of determining

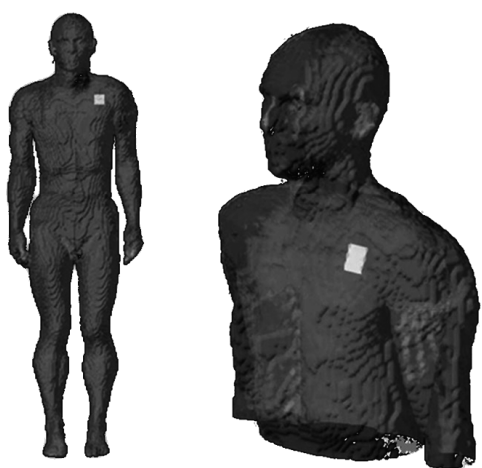

Fig. 7 Representation of the phantom model with pacemaker.

Table 2 Maximum interference distance for frequency used.

\begin{tabular}{c|c}
\hline Frequency & Maximum Interference Distance \\
\hline $900 \mathrm{MHz}$ & $16 \mathrm{~cm}$ \\
$1500 \mathrm{MHz}$ & $6 \mathrm{~cm}$ \\
$2100 \mathrm{MHz}$ & $2 \mathrm{~cm}$ \\
\hline
\end{tabular}

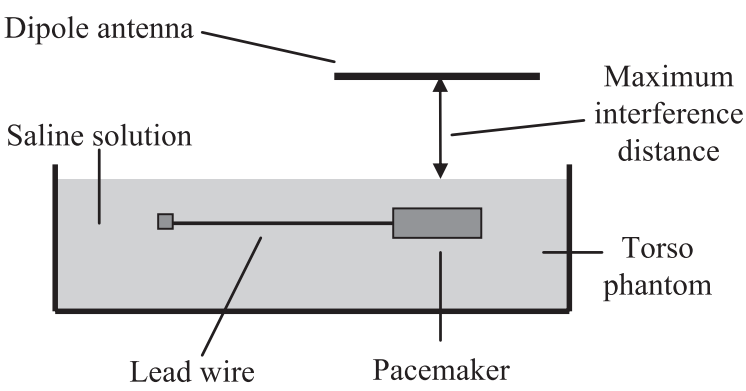

Fig. 8 Representation of experimental test system.

whether the field strengths are within the safe limits for pacemaker operation, it is necessary to normalize the values against a reference level that represents the threshold values for each frequency. In this paper, maximum interference distance, the experimental results of pacemaker immunity tests against cellular radios conducted in [11], are used for pacemaker EMI estimation. These distances are shown in Table 2. Figure 8 shows the pacemaker immunity tests system. Previously, these distances were used in Ref. [10] to calculate the reference values $(0 \mathrm{~dB}$ point $)$ for a dipole antenna in free-space, that is substituted an EMI test without pacemaker and torso model. The calculations were made in the direction of the plane perpendicular to the antenna axis.

In the proposing method, the reference values were calculated by simulation for the experimental test system shown in Fig. 8. The dipole antenna is set at maximum interference distance from torso phantom for each frequency. The value of electric field strength near the pacemaker is used as the reference value and is plotted in the histograms as the $0 \mathrm{~dB}$ point. Any deviation (increase) in the electric field strength values from this reference indicates the likelihood of pacemaker malfunction. Figure 9 shows the flowchart of pacemaker EMI estimation. 


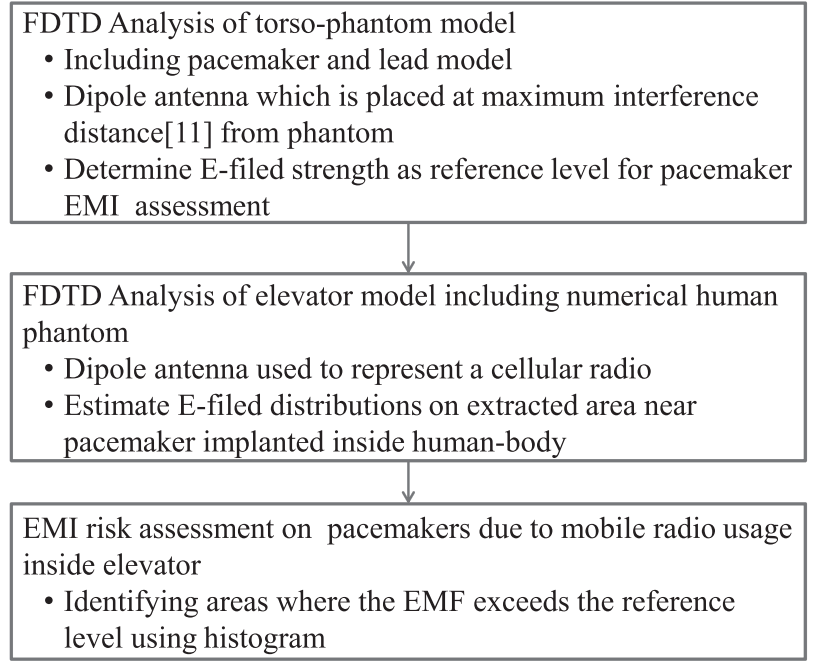

Fig. 9 Flowchart for estimating pacemaker EMI.

\section{E-field Histograms Differences between Previous Method and Proposed Method}

This section determines the differences in the E-field histograms created by the previous method and the proposed method for a single user standing at the elevator.

\subsection{E-field Histograms Inside Elevator}

The histograms yielded by the previous method are shown Fig. 10. The $x$ axis denote the relative E-field strength. The $y$ axis denote the ratio of cells at each class to all cells included in evaluation area. The E-field values were normalized to the reference value obtained using the maximum interference distance in free-space. It is represented on the histograms by the $0 \mathrm{~dB}$ point.

The maximum estimated values are with in the range of " $-1 \mathrm{~dB}$ to $0 \mathrm{~dB}$," " $-5 \mathrm{~dB}$ to $-4 \mathrm{~dB}$," and " $-11 \mathrm{~dB}$ to $-10 \mathrm{~dB}$ " for the frequencies of $900 \mathrm{MHz}, 1500 \mathrm{MHz}$, and $2100 \mathrm{MHz}$, respectively. The rations of maximum E-field strengths at each frequency were $0.28 \%, 0.03 \%$, and $0.07 \%$ respectively. As the frequency increases, the peak values of E-field strength decreases further. This is because the maximum interference distance shortens as the frequency increases.

\subsection{E-field Histograms Inside the Region of Pacemaker Implant}

This section introduces the histograms created by the proposed method. The user is located at the center of the elevator. Figure 11 shows the E-field histograms for the 3 frequencies examined. The histograms were derived from electric field strength values in the region of interest as shown in Fig. 5. The E-field values were normalized to the reference value obtained using dipole antenna and pacemaker model inside torso model. The maximum values are with in the range of " $-6 \mathrm{~dB}$ to $-4 \mathrm{~dB}$," " $-12 \mathrm{~dB}$ to $-10 \mathrm{~dB}$,"

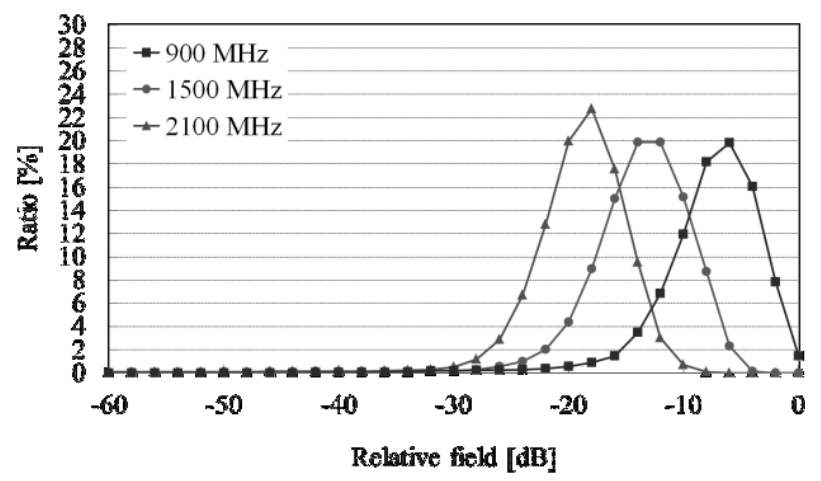

Fig. 10 E-field histograms at the height of $130 \mathrm{~cm}$ inside the elevator for each frequency.

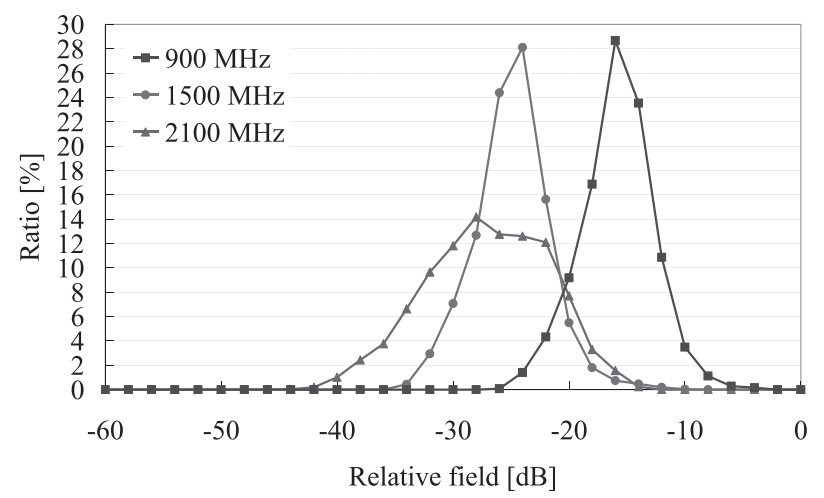

Fig. 11 E-field histograms of the region of interest.

and " $-14 \mathrm{~dB}$ to $-12 \mathrm{~dB}$ " for the frequencies of $900 \mathrm{MHz}$, $1500 \mathrm{MHz}$, and $2100 \mathrm{MHz}$, respectively. The rations of maximum E-field strengths at each frequency were $0.16 \%$, $0.04 \%$, and $0.24 \%$ respectively.

From Fig. 10 and Fig. 11, the difference of maximum E-field strength between the 2-dimensional E-fielsd distributions in the elevator and E-field distributions in region of interest is approximately $4 \mathrm{~dB}, 6 \mathrm{~dB}$, and $2 \mathrm{~dB}$ for the frequencies of $900 \mathrm{MHz}, 1500 \mathrm{MHz}$, and $2100 \mathrm{MHz}$, respectively. For all 3 frequencies, the histograms of the region of interest are lower than those on the horizontal plane.

\subsection{E-field Histograms Using Homogeneous Phantom Model}

In this section, the differences in the E-field histogram between homogeneous and inhomogeneous phantom models, were investigated. Figure 12 shows the E-field histograms for homogeneous phantom models examined under same condition of Fig. 11. From Fig. 11 and Fig. 12, there are not much differences in frequency characteristics. However, the maximum E-field strengths for homogeneous phantom models were about $3 \mathrm{~dB}$ lower than those for inhomogeneous models for all frequencies carried out. From these results, the estimation using inhomogeneous phantom model are thought to be conservative. 


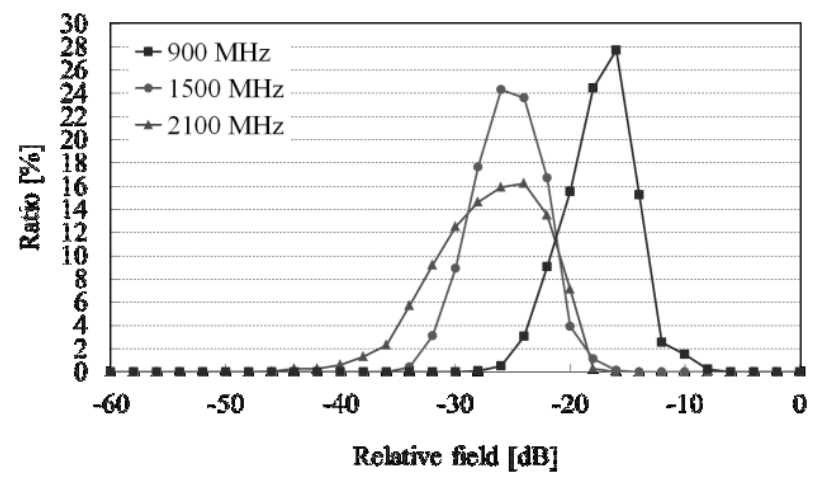

Fig. 12 E-field histograms using homogeneous phantom model.

\section{EMI Estimation Results}

4.1 Variation in Field Strength as the User Position Changes

In order to investigate whether or not there are certain positions in the elevator where implantable cardiac pacemakers are more susceptible to EMI, human phantom model is placed in center or corner of the elevator as a user. Figure 13 show the variation in E-field for the 3 frequencies being carried out.

It is observed that the differences in maximum Efield strength with this change in position are about $2 \mathrm{~dB}$, $6 \mathrm{~dB}$, and $2 \mathrm{~dB}$ for the frequencies $900 \mathrm{MHz}, 1500 \mathrm{MHz}$, $2100 \mathrm{MHz}$, respectively.

4.2 Comparison of the Presence or Absence of a Pacemaker

This section determines the impact of adding the pacemaker to the phantom model. Figure 14 shows the variation of Relative field strength in the region of interest with and without the pacemaker when the user is located at the center of the elevator for the three frequencies.

For all 3 frequencies, it is observed that the pacemaker disrupts the propagating electromagnetic waves and lowers the relative field density. The maximum E-field values with pacemaker are within the range of " $-6 \mathrm{~dB}$ to $-4 \mathrm{~dB}$," " $-14 \mathrm{~dB}$ to $-12 \mathrm{~dB}$," and " $-16 \mathrm{~dB}$ to $-14 \mathrm{~dB}$ " for the frequencies of $900 \mathrm{MHz}, 1500 \mathrm{MHz}$, and $2100 \mathrm{MHz}$, respectively. However, there is not much difference in the maximum E-field strength. Differences of the maximum E-field strength are less than $2 \mathrm{~dB}$.

\section{Discussion and Conclusion}

This paper presented a detailed investigation of pacemaker EMI in the elevator. EMI impact in the elevators was estimated using precise numerical analyses and an anatomically-correct human phantom model at the three mobile phone frequency bands.

We proposed a method for EMI impact estimation. The

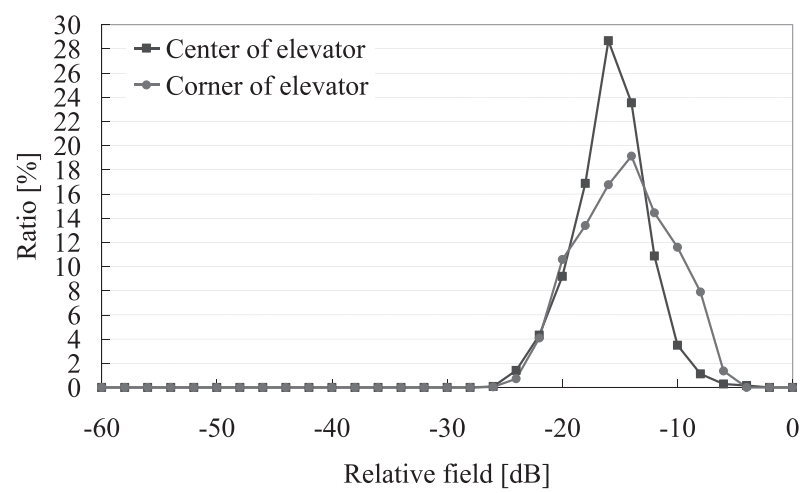

(a)

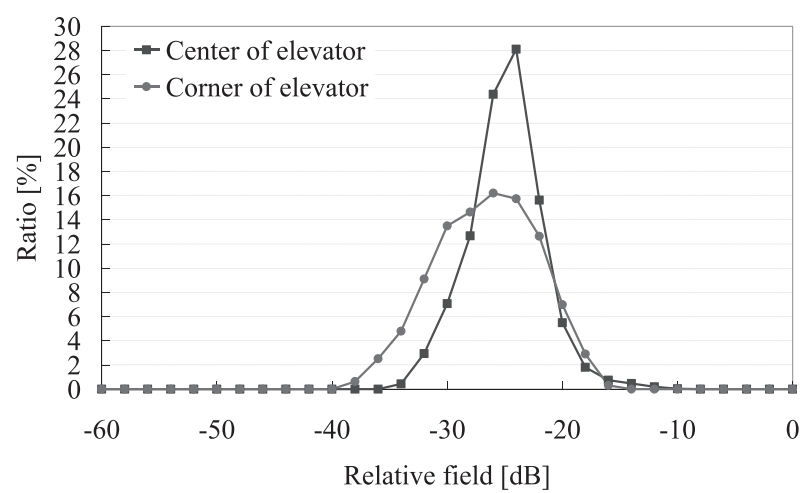

(b)

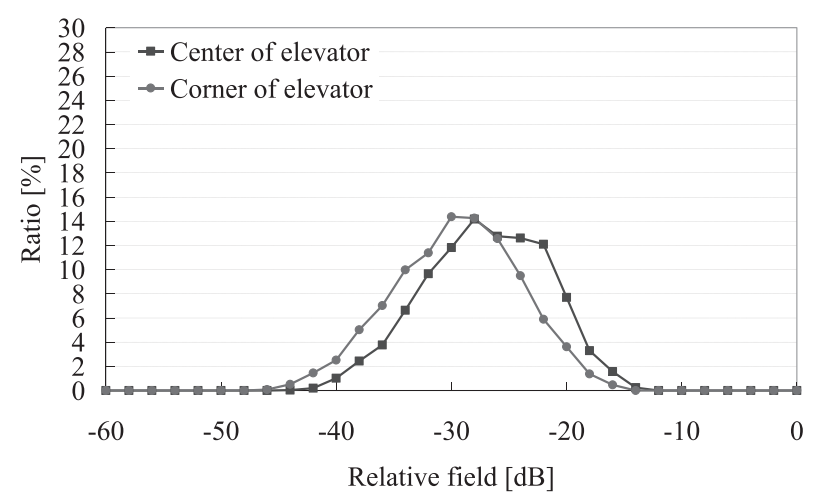

(c)

Fig. 13 Variation in E-field as the position of the human models changes for frequencies (a) $900 \mathrm{MHz}$, (b) $1500 \mathrm{MHz}$, and (c) $2100 \mathrm{MHz}$.

E-field strengths in the region of pacemaker implant were examined by comparing E-field strengths with reference values obtained by using the maximum interference distance of implantable cardiac pacemakers. It is therefore shown that EMI impact on implantable cardiac pacemakers can be precisely assessed using inhomogeneous human phantom model based on proposed method.

The results presented in Sect. 4 indicate that, for $900 \mathrm{MHz}, 1500 \mathrm{MHz}$ and $2100 \mathrm{MHz}$ bands carried out, calculated E-field strengths were sufficiently low to trigger pacemaker malfunction. The maximum E-field strengths 


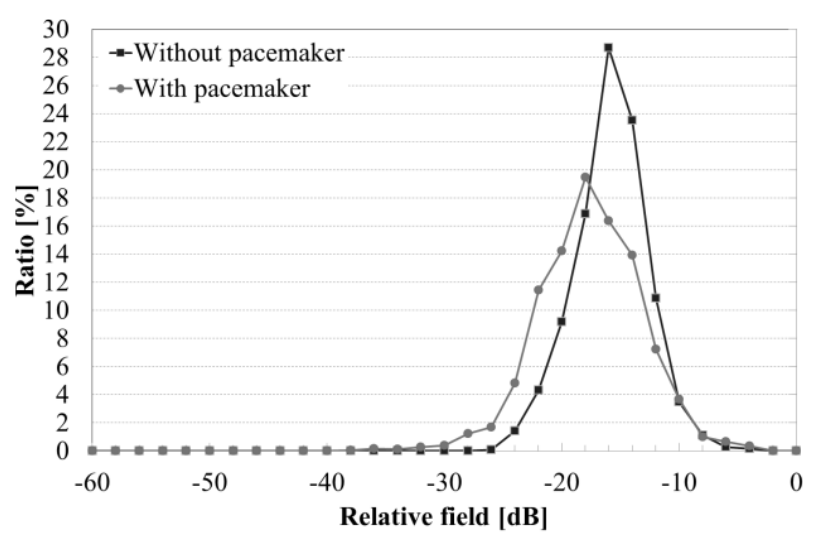

(a)

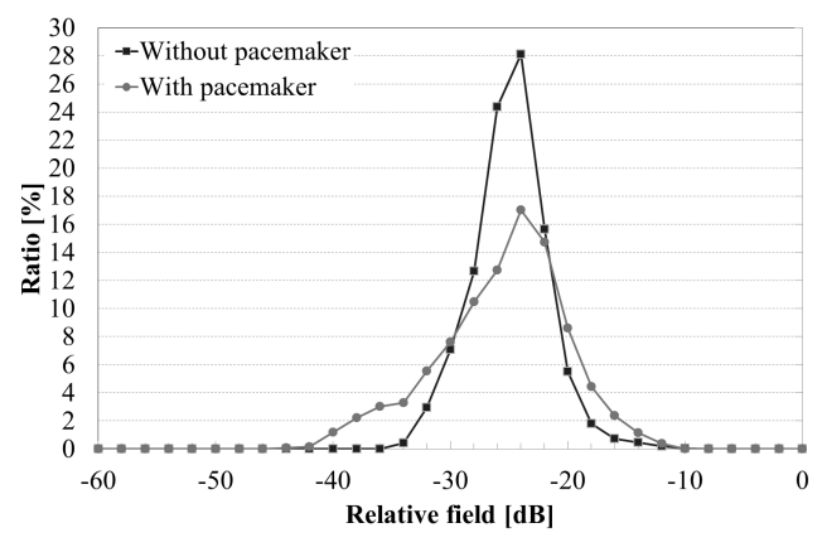

(b)

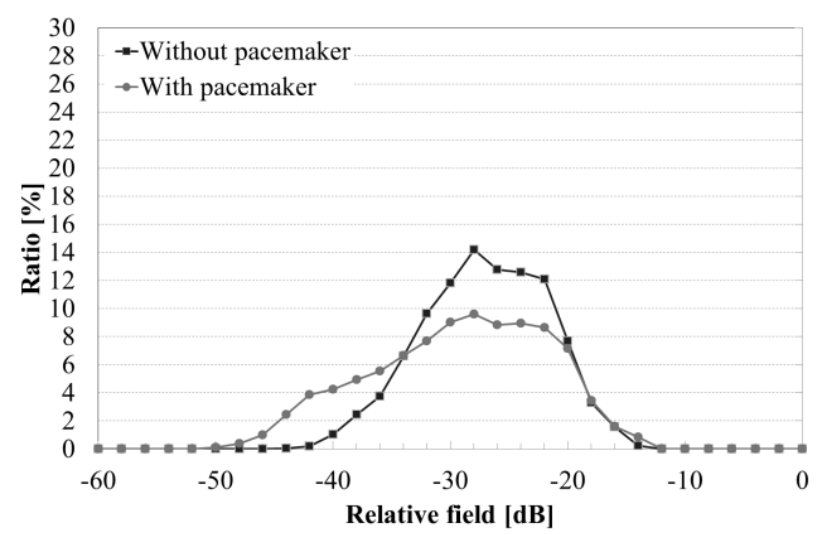

(c)

Fig. 14 E-field histograms of with or with out pacemaker for frequencies (a) $900 \mathrm{MHz}$, (b) $1500 \mathrm{MHz}$, and (c) $2100 \mathrm{MHz}$.

were found to vary with the phantom position in the elevator. There is not much difference in the maximum Efield strengths between with and without a pacemaker. Furthermore, the differences in the maximum E-field strengths while using homogeneous and using inhomogeneous phantom models is about $3 \mathrm{~dB}$. Therefore, we can conclude that pacemaker EMI due to cellular radio in elevator can be conservatively estimated using inhomogeneous phantom model.

\section{Acknowledgments}

This study was partially supported by the Electromagnetic Environment Committee of Association of Radio Industries and Businesses (ARIB) in JAPAN.

\section{References}

[1] A. Kramer, J. Frohlich, and N. Kuster, "Towards danger of mobile phones in planes, trains, cars and elevators," J. Physical Society Japan, vol.71, 3100, Dec. 2002.

[2] H. Hondo, "Rising level of public exposure to mobile phones," J. Physical Society of Japan, vol.71, pp.432-435, Feb. 2002.

[3] H. Dominguez, A. Raizer, and W.P. Carpes, Jr., "Electromagnetic fields radiated by a cellular phone in close proximity to metallic walls," IEEE Trans. Magn., vol.38, no.2, pp.793-796, March 2002.

[4] M.P. Robinson, J. Clegg, and A.C. Marvin, "Radio frequency electromagnetic fields in large conducting enclosure: Effects of apertures and human bodies on propagation and field-statistics," IEEE Trans. Electromagn. Compat., vol 48, no.2, pp.304-310, May 2006.

[5] V. Barbaro, P. Bartolini, A. Donato, and C. Militello, "Electromagnetic interference of analog cellular telephone with pacemakers," J. Pacing and Clinical Electrophysiology, vol.19, no.10, pp.14101418, Oct. 1996.

[6] W. Irnich, L. Batz, R. Muller, and R. Tobisch, "Electromagnetic interference of pacemakers by mobile phones," J. Pacing and Clinical Electrophysiology, vol.19, no.10, pp.1431-1446, Oct. 1996.

[7] D.L. Hayes, P.J. Wang, D.W. Reynolds, M. Estes, III, J.L. Griffith, R.A. Steffens, G.L. Carlo, G.K. Findlay, and C.M. Johnson, "Interference with cardiac pacemakers by cellular telephones," New Engl. J. Med., vol.336, no.21, pp.1473-1479, May 1997.

[8] K. Kunz and R. Luebbers, The Finite Difference Time Domain Method for Electromagnetics, CRC Press, 1993.

[9] T. Hikage, L. Harris, T. Nojima, S. Ally, and S. Watanabe, "Estimations for implantable cardiac pacemakers EMI from cellular radios in narrow space multi-reflection environments," Proc. 2008 AsiaPacific EMC Symposium, pp.128-131, May 2008.

[10] L. Harris, T. Hikage, and T. Nojima, "Precise estimation of cellular radio electromagnetic field in elevators and EMI impact on implantable cardiac pacemakers," IEICE Trans. Electron., vol.E92-C, no.9, pp.1182-1187, Sept. 2009.

[11] "Results of Investigation into the effects of electromagnetic waves on medical equipment," Association of Radio Industries and Business (ARIB) Japan, March 2001-2003, 2006. Available: e.g. http://www.soumu.go.jp/s-news/2002/020702_3_1.html

[12] T. Hikage, T. Nojima, S. Watanabe, and T. Shinozuka, "Electric-field distribution estimation in a train carriage due to cellular radios in order to assess the implantable cardiac pacemaker EMI in semi-echoic environments," IEICE Trans. Commun., vol.E88-B, no.8, pp.32813286, Aug. 2005.

[13] J.P. Berenger, "A perfect matched layer for the absorptions of electromagnetic waves," J. Comput. Phys., vol.114, no.2, pp.185-200, Oct. 1994.

[14] A. Kitagawa, T. Hikage, T. Nojima, A. Simba, and S. Watanabe, "Large scale FDTD analysis for the electromagnetic field distribution estimations in elevator using precise numerical phantom model," Proc. 2009 International Symposium on Electromagnetic Compatibility, pp.517-520, Kyoto, July 2009.

[15] A. Christ, W. Kainz, E.G. Hahn, K. Honegger, M. Zefferer, E. Neufeld, W. Rascher, R. Janka, W. Bautz, J. Chen, B. Kiefer, P. Schmitt, H.P. Hollenbach, J.X. Shen, M. Oberle, and N. Kuster, The Virtual Family - Development of anatomical CAD models of two adults and two children for dosimetric simulations.

[16] C. Gabriel, "Compilation of the dielectric properties of body tissues 
at RF and microwave frequencies," Brooks Air Force Technical Report AL/OE-TR-1996-0037, 1996.

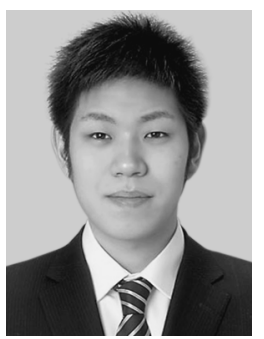

Atsushi Kitagawa received the B.E. degree in electronics and information engineering from Hokkaido University, Sapporo, Japan, in 2008. $\mathrm{He}$ is currently working toward the M.E. degree at the Graduate School of Information Science and Technology, Hokkaido University. His research interests are FDTD analysis and EMC issues. He is a student member of the IEEE.

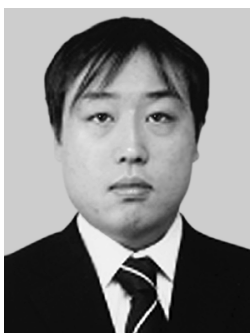

Takashi Hikage

received the B.E., M.E. and $\mathrm{Ph} . \mathrm{D}$. degrees in electronics and information engineering from Hokkaido University, Sapporo, Japan, in 1997, 1999 and 2002, respectively. From 1999 to 2003, he was with the Graduate School of Engineering, Hokkaido University. Now, he is an Instructor of the Graduate School of Information Science and Technology, Hokkaido University, Sapporo. His research interests are FDTD analysis and EMC issues. Dr. Hikage is a member of the IEEE.

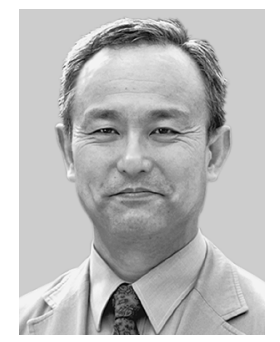

Toshio Nojima received a B.E. in electrical engineering from Saitama University, Japan in 1972 and received M.E. and Ph.D. degrees in electronic engineering from Hokkaido University in 1974 and 1988. From 1974 to 1992 he was with Nippon Telegraph and Telephone (NTT) Communications Laboratories, where he was engaged in the development of highcapacity microwave radio relay systems. From 1992 to 2001 he was with the Research Laboratory of NTT DoCoMo in Yokosuka, where he was a Senior Executive Research Engineer and conducted research on radio safety and EMC issues related to mobile radio systems as well as microwave circuit technologies. Since January of 2002 he has been a professor at the Graduate School of Information Science and Technology, Hokkaido University, Sapporo. Dr. Nojima is a member of the IEEE, the Institute of Electrical Engineers of Japan, and the Bioelectromagnetics Society.

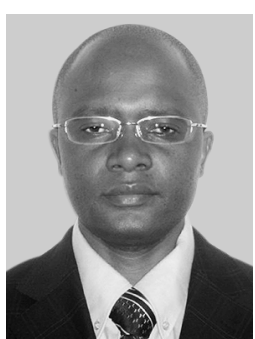

Ally Y. Simba was born in Bagamoyo, Tanzania. He received the B.Sc. degree (with honors) in electronics science and communication from the University of Dar es salaam, Dar es Salaam, Tanzania, in 1998, and the M.E. and Ph.D. degrees in electronics and information engineering from Hokkaido University, Sapporo, Japan, in 2003 and 2006. In 2006, he joined the Biomedical Electromagnetic Compatibility (EMC) Group, National Institute of Information and Communications Technology, Tokyo, Japan, as an Expert Researcher. His recent research interest is bioelectromagnetics. Dr. Simba is a member of the Institute of Engineering and Technology (IET), U.K. He was the recipient of the Japanese Government Scholarship (Monbusho Scholarship) (2000-2006)

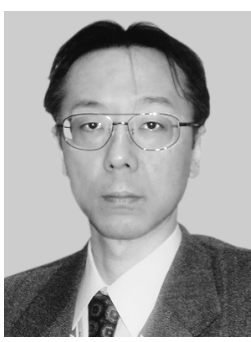

Soichi Watanabe

received the B.E. M.E., and D.E. degrees in electrical engineering from Tokyo Metropolitan University, Tokyo, Japan, in 1991, 1993, and 1996, respectively. He is currently with the National Institute of Information and communications Technology (NICT), Tokyo, Japan. His main research interest is biomedical electromagnetic compatibility. Dr. Watanabe is a member of the Institute of Electrical Engineers (IEE), Japan, and the Bioelectromagnetics Society. Since 2004, he has also been a member of the Standing Committee on Physics and Engineering of the ICNIRP. He was the recipient of several awards, including the 1996 International Scientific Radio Union (URSI) Young Scientist Award and the 1997 IEICE Best Paper Award. 